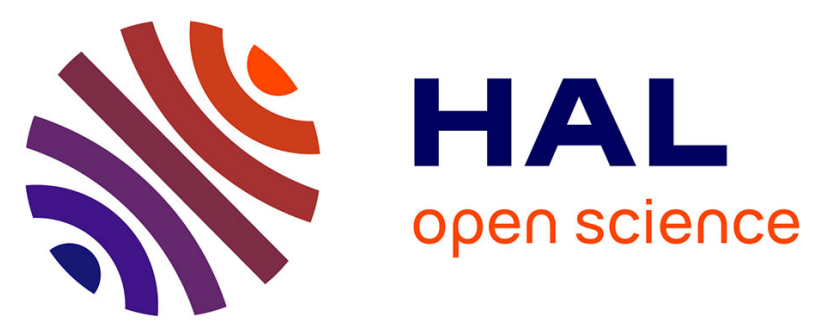

\title{
Tolerance analysis of overconstrained and flexible assemblies by polytopes and finite element computations: application to a flange
}

Doriane Gouyou, Yann Ledoux, Denis Teissandier, Vincent Delos

\section{- To cite this version:}

Doriane Gouyou, Yann Ledoux, Denis Teissandier, Vincent Delos. Tolerance analysis of overconstrained and flexible assemblies by polytopes and finite element computations: application to a flange. Research in Engineering Design, 2017, 10.1007/s00163-017-0256-5 . hal-01515475

\author{
HAL Id: hal-01515475 \\ https://hal.science/hal-01515475
}

Submitted on 27 Apr 2017

HAL is a multi-disciplinary open access archive for the deposit and dissemination of scientific research documents, whether they are published or not. The documents may come from teaching and research institutions in France or abroad, or from public or private research centers.
L'archive ouverte pluridisciplinaire HAL, est destinée au dépôt et à la diffusion de documents scientifiques de niveau recherche, publiés ou non, émanant des établissements d'enseignement et de recherche français ou étrangers, des laboratoires publics ou privés. 


\title{
Tolerance analysis of overconstrained and flexible assemblies by polytopes and finite element computations: application to a flange
}

\author{
Doriane Gouyou* Yann Ledoux \\ Vincent Delos
}

Denis Teissandier

April 27, 2017

\begin{abstract}
In industrial contexts, overconstrained assemblies are often used to ensure sufficient stiffness and accuracy of assembly. Such architectures are quite usual, however, analysis and synthesis of the tolerance are not easy to define and quantify. In these cases, the compliance of the assembly is not automatic and deformations may often occur, requiring a particular and difficult analysis of the assembly from scientific and computing points of view.

The present work addresses such overconstrained mechanisms through a general and a sequential approach. Based on this, it is possible to determine the final assembly condition (with or without interference) as a function of part defects. First, the assembly procedure is performed based on polytope computations, assuming a rigid part behavior. From this, a stochastic simulation is performed and some non-compliant assemblies (assemblies with interferences) are identified. For these assemblies, the rigid behavior of parts is then overcome by means of finite element simulations and a typical procedure is set up to introduce part defects. We then deduce whether the assembly can be made from the load needed to assemble the parts. This procedure is applied to a flange composed of five pin / hole pairs, as this is a highly overconstrained mechanism.
\end{abstract}

\section{Nomenclature}

- $t_{M-1, i / 2, i}$ : translation vector of surface $\mathrm{i}$ of part 1 in relation to surface $\mathrm{i}$ of part 2 at point $M$. If $i=0$, we are considering the translation of the nominal geometry of part 1 in relation to the nominal geometry of part 2 at point $\mathrm{M}$.

- $r_{1, i / 2, i}$ : rotation vector of surface $\mathrm{i}$ of part 1 in relation to surface $\mathrm{i}$ of part 2 . If $i=0$, we are considering the rotation of the nominal geometry of part 1 in relation to the nominal geometry of part 2 ,

- $e_{M-1, i / 1,0}$ : location defect vector of surface $i$ of part 1 in relation to its nominal geometry at point $M$,

- $e_{M-2, i / 2,0}$ : location defect vector of surface $i$ of part 2 in relation to its nominal geometry at point $M$, 
- $D_{n}, D_{1, i}$ and $D_{2, i}$ : the diameter of the nominal surface, the diameters of surface i of part 1 and surface $i$ of part 2 respectively,

- $\bar{H}_{i, j}^{-}$: half-space of the contact constraint derived from the $j^{\text {th }}$ discretization point between surface $i$ of part 1 and surface $i$ of part 2,

- $P_{i}$ : contact polytope of surface $\mathrm{i}$ of part 1 in relation to surface $\mathrm{i}$ of part 2,

- $P_{R}$ : resulting contact polytope of part 1 in relation to part 2 .

\section{Introduction}

Tolerance analysis consists in simulating the behavior of a mechanical system based on the consideration of part defects. To perform such an analysis, it is crucial not only to consider individual parts but also parts interacting one with another.

The phenomenon of interaction between parts becomes prominent when mechanisms correspond to overconstrained assemblies. On an industrial scale, overconstrained assemblies are often used. The main advantage of this type of assembly is the increased stiffness of the mechanical joints and thus the limitations of deviations in parts subject to external mechanical loads. This is mainly due to the redundancy of contacts between parts. As a consequence, the assembly of parts can be uncertain and two different scenarios may occur: either the parts are assembled without any interference, or some interference between surfaces of parts can be identified. In such cases, following the classical tolerance hypothesis (i.e. no part deformation), the assembly is assumed to be impossible. For small relative interference values regarding the stiffness of the assembly, the compliancy of the parts could make the assembly possible with local modifications to part geometry, leading to a residual stress state in the parts. Tolerance analysis of the overconstrained mechanisms is not an easy task, since the contact between parts can evolve as a function of a local defect of parts. This requires a more complex algorithm and associated numerical tools solely for methods which assume classical tolerance study hypotheses (rigid behavior of part, no form defects of contact surfaces). In the case of coupling the deformations of parts and the tolerance study, considerable non-linearity appears, mainly due to the evolution of the contact zones between the parts as a function of interference locations and values. This scientific bottleneck is one of the major contributions of this paper and to the best of our knowledge, no commercial or research software is available to assist people in industry to quantify and synthesize an assembly according to part defects.

To make the approach more tractable and comprehensive, it is applied to a typical overconstrained mechanism, as shown in Section 3.1, composed of two parts. This architecture is derived from industrial applications. Using geometrical specifications, the tolerance analysis is performed by means of polytope computations derived from the resolution of inequalities. These inequalities aim at translating geometrical specifications of parts into the ability to assemble the parts that make up the mechanism. To simulate assembly with defects, stochastic simulations are performed assuming rigid behavior of parts (Section 3.3). According to the geometrical specifications, local interferences are identified between parts and should result in making the assembly im- 
possible. From the developed polytope computation, it is possible to clearly identify the interference values and their location on the parts. Assuming part deformations and hence interferences, it is proposed to rank the difficulty in performing the assembly. To carry out this quantification, a finite element simulation is used and an equivalent load is estimated to assemble and deform parts. The influence of the interference values on the load required to assemble the mechanism is tested by a parametric study. Through a linear evolution of the interference values, a drastic evolution of the contact pressure and contact location can be observed, leading to an exponential increase in the assembly load.

\section{Tolerance study of overconstrained assembly}

The tolerance methods and associated tools available in the literature are mainly related to the architecture of the product. In the case of an isostatic mechanism, analysis of the product behavior from the geometric point of view is relatively simple and the final position of one part in relation to the other could be directly linked to the accumulation of defects from parts. In this way, the accuracy of the mechanism could be determined. Classical approaches based, for instance, on small displacement fields (Bourdet et al, 1996; Clément and Bourdet, 1988) could be relevant. To illustrate such an approach, the study by Ledoux and Teissandier (2013) on the clearance between the blades and shaft of a turboshaft engine could be cited. In this study the statistical analysis leads to a probabilistic aspect to quantify the risk of contact between the blade and the shaft.

In Nigam and Turner (1995), the authors are more focused on the selection of the most relevant statistical methods for the tolerance analysis. Recently, commercial software packages have been developed to assist engineers in this task. To cite some of them, MECAmaster (MECAmaster SARL, 2012), 3DCS (DCS, 2011) or CLIC (Anselmetti, 2010) could be used for $1 D$ or 3D applications. Statistical tools have been included in these software packages to relate the part defects to functional geometrical requirements. According to Shah et al (2007), none of these tools are able to consider overconstrained mechanisms. Indeed, in overconstrained mechanisms, it is more complex to determine the position of parts since gaps are present in joints, allowing local part displacements. Basically, new functional constraints appear and the tolerance analysis should validate both the respect of functional conditions (i.e. position between parts and gap values) and the fact that the assembly of parts could be possible. More advanced formulation of the problem is then required.

One such formulation consists in using sets of constraints, a concept introduced by Fleming (1988). Several studies have been carried out using this principle: these include feasibility spaces (Turner, 1993), T-Maps (Davidson et al, 2002), Domains (Giordano et al, 1992), and Polytopes (Teissandier et al, 1999). There are also articles comparing some of these tolerance analyses (Mansuy et al, 2013a; Ameta et al, 2011). T-Maps and domains can be used in worst case or statistical approaches (Ameta et al, 2007; Mansuy et al, 2013b). Constraint satisfaction approaches have been developed based on simulated populations of parts using the Monte Carlo method. These studies ensure the compliance of contact stresses in overconstrained mechanisms using linear optimization methods combined with reliability calculation algorithms (Dantan and 
Qureshi, 2009; Beaucaire et al, 2013). Another problem related to tolerance study is the consideration of part deformations. Due to external loads applied to the mechanism, the final position of parts may vary according to their own stiffness, local deformations of contact surfaces and possible movements in the mechanical joint induced by the local gaps. A few studies related to isostatic mechanism could be cited like Shiu et al (2003) for static mechanisms, Soderberg et al (2006) and Cid et al (2007) proposing deformations of several parts, Xie et al (2007) and Hu and Camelio (2006) including the influence of the assembly sequence and the part deformations or Jaishankar et al (2012, 2013) using T-Map models. In the studies by Chevassus et al (2006), Breteau et al (2007) and Mounaud et al (2007), part deformations are introduced for overconstrained applications. Domain computation modeling the stiffness of mechanical joints has been proposed by Samper and Giordano (2003) and Pierre et al (2014). Some authors have included deformation in polytope computations. However, these studies do not deal with assembly interferences.

Finally, in Pezzuti et al (2005), the authors have studied a fairly similar application to the architecture proposed in the present paper. It is composed of an assembly of two pins and holes. Deformations are only assumed in the local contact of pins and holes, while the global body of the part is rigid; the position and orientation defects of both pins and holes are considered. The compliant assembly rate is then quantified through a hypothesis of rigid parts (without any deformation) and local deformations. In the present study, it is proposed to extend this application by means of polytope formulation to improve the consideration of 3D geometric defects. A statistical hypothesis derived from industrial knowledge is used to quantify the compliant assembly rate.

\section{Assembly simulation}

\subsection{Studied mechanism}

To illustrate the general approach developed in the present paper, we applied it to a mechanism which corresponds to a flange made up of two parts (parts 1 and 2 respectively) as shown in Fig.1. The joint is composed of a planar pair joint added to five Ball-and-Cylinder pair joints held by pins (supported by part 1) and holes (belonging to part 2) distributed around a circle. The joint between a pin and a hole is modeled by a Ball-and-Cylinder pair joint since the holes are short in length. This type of architecture corresponds to a highly overconstrained mechanism, and is often used in aeronautics, space, nuclear physics, etc. Usually the parts are clamped by bolts, but these components are not considered in this study. The definition of geometric specifications could be determined through different approaches, according to the requirement on the compliant assembly rate. If it is expected that all assemblies are compliant, it is then proposed that computations are run following the worst case hypotheses. This leads to small geometric specification values (increasing the manufacturing cost). If a small percentage of non-compliant assemblies is allowed, stochastic approaches could be relevant and be used to define a tradeoff between geometric specification values and manufacturing costs. In the present work, the geometric specification has been defined through another approach. Knowing the manufacturing procedure and the means used 


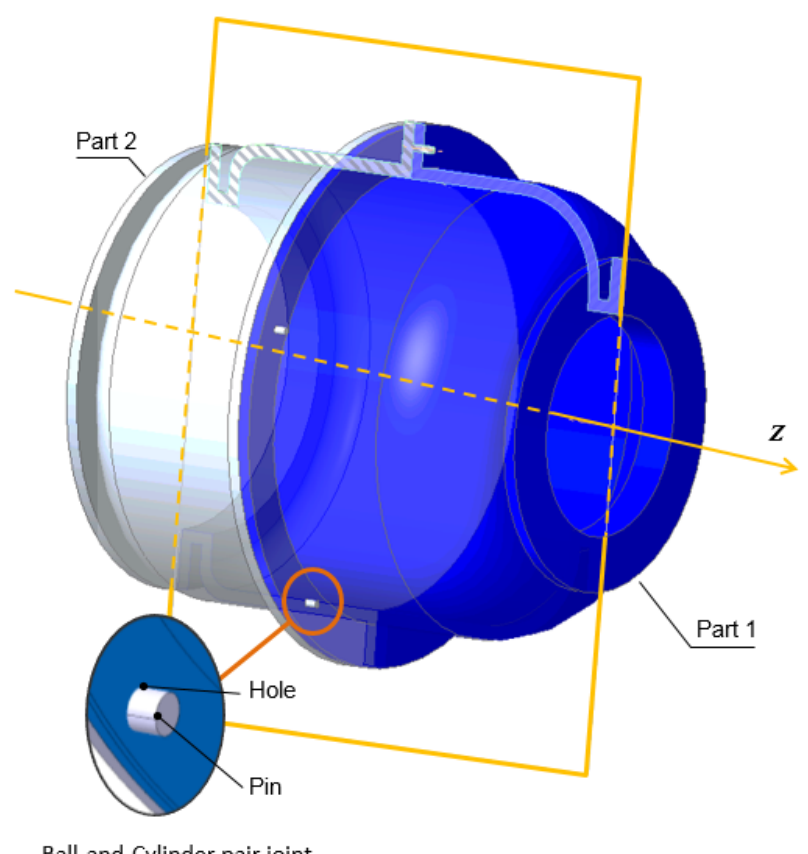

Ball-and-Cylinder pair joint

Figure 1: Flange-type joint and associated geometry

to produce parts, the capability of these means has been quantified by our industrial partner. From these values, the geometric specification values have been deduced. The technical drawings of the parts are shown in Fig. 2.

\subsection{Geometric parameters and definition of part defects}

Fig. 3 details the different contact surfaces between the parts. The real surfaces of the parts are modeled by substituted surfaces (ISO5459, 2011). Surfaces $1, i$ and 2,i ( $i \in\{1, \ldots, 5\}$ ) have a cylindrical shape and surfaces 1,6 and 2,6 are planes. In the nominal configuration defined in $\mathrm{CAD}$, the axes of the cylindrical surfaces are parallel to the nominal axis of the flange (called Flange plane in Fig. 3) and all of them are distributed at uniform angles (one pin and hole every $72^{\circ}$ ).

Fig. 4 details the geometric parameters used for this study. Let $R_{0}(O, \vec{x}, \vec{y}, \vec{z})$ be the reference system associated with the nominal geometry of the parts. Points $N_{0 i}$ are the respective nominal positions of the pin and hole pairs in the flange plane. The origin $\mathrm{O}$ of reference system $R_{0}$ is the center of the circle $C e_{0}$ with radius $R_{t h}$ passing through points $N_{0 i}(i \in\{1, \ldots, 5\})$ and the axis $O \vec{x}$ passes through the point $N_{01}$. The different values for every geometric parameter are given in Table 1.

The part defects are assumed to be held in the flange plane $(\vec{x}, \vec{y})$ and are expressed through deviation projections along the $\vec{x}$ and $\vec{y}$ axes around the nominal positions, as 

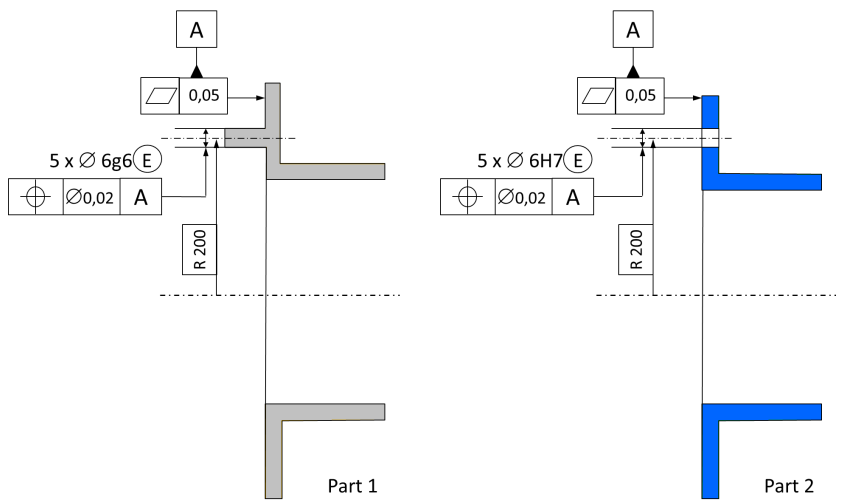

Figure 2: Definition of parts and geometric specifications

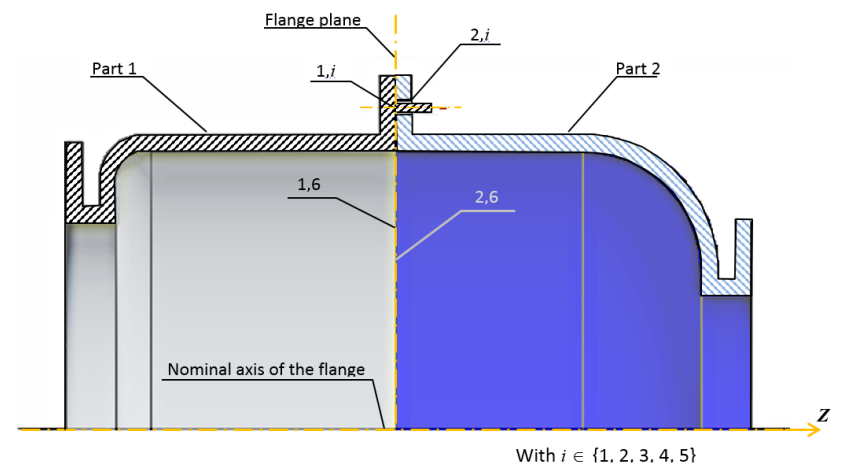

Figure 3: Substituted surfaces

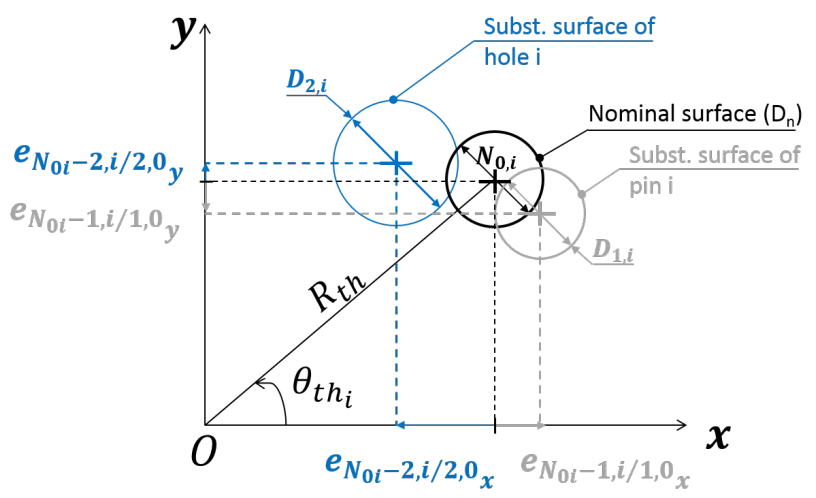

Figure 4: Parameters used to characterize the position defects of simulated pins and holes 
Table 1: Nominal geometry parameters

\begin{tabular}{lll}
\hline Theoretical radius of pins and holes location & $R_{t h}$ & $200 \mathrm{~mm}$ \\
\hline Theoretical angles of pins and holes location & $\theta_{t h_{i}}$ & every $72^{\circ}$ \\
\hline Nominal diameter of pins and holes & $D_{n}$ & $6 \mathrm{~mm}$ \\
\hline
\end{tabular}

Table 2: Simulated geometry parameters

\begin{tabular}{lll}
\hline \multirow{2}{*}{ Pins } & Simulated diameters & $D_{1, i}$ \\
\cline { 2 - 3 } & Location deviations along $\vec{x}$ at $N_{0 i}$ & $e_{N_{0 i}-1, i / 1,0_{x}}$ \\
\cline { 2 - 3 } & Location deviations along $\vec{y}$ at $N_{0 i}$ & $e_{N_{0 i}-1, i / 1,0_{y}}$ \\
\hline \multirow{3}{*}{ Holes } & Simulated diameters & $D_{2, i}$ \\
\cline { 2 - 3 } & Location deviations along $\vec{x}$ at $N_{0 i}$ & $e_{N_{0 i}-2, i / 2,0_{x}}$ \\
\cline { 2 - 3 } & Location deviations along $\vec{y}$ at $N_{0 i}$ & $e_{N_{0 i}-2, i / 2,0_{y}}$ \\
\hline
\end{tabular}

shown in Fig. 4. The list of geometric parameters is given in Table 2.

Geometric defects are generated by the Monte Carlo method according to Gaussian statistical distribution laws for the diameters of pins and holes, and for the location deviations of the centers of the pins and holes. The estimate of the standard deviation for the Gaussian law is determined as $\sigma=g s / 6$ (such that $g s$ corresponds to either the location specification or the tolerance associated to diameters of pins and holes). All the distributions are centered on theoretical locations and diameters. These characteristics are:

- Distribution of pins:

- For diameters: $N(5.992 \mathrm{~mm}, 1.3 \mu \mathrm{m}) \rightarrow D_{1, i}$

- For location deviations:

* Along $x-\vec{a}$ axis, normal distribution $N(0 m m, 3.3 \mu \mathrm{m}) \rightarrow e_{N_{0 i}-1, i / 1,0_{x}}$

* Along $y-\overrightarrow{a x i s}$, normal distribution $N(0 m m, 3.3 \mu m) \rightarrow e_{N_{0 i}-1, i / 1,0_{y}}$

- Distribution of holes:

- For diameters: $N(6.006 m m, 2 \mu m) \rightarrow D_{2, i}$

- For location deviations:

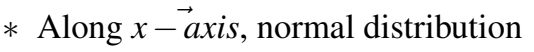
$N(0 m m, 3.3 \mu m) \rightarrow e_{N_{0 i}-2, i / 2,0_{x}}$

* Along $y-\overrightarrow{a x i s}$, normal distribution $N(0 \mathrm{~mm}, 3.3 \mu \mathrm{m}) \rightarrow e_{N_{0 i}-2, i / 2,0_{y}}$ 


\subsection{Assembly simulations of rigid parts}

The assembly procedure developed in this section assumed a rigid behavior of parts and no form defect of contact surfaces. Following these hypotheses, the compliance of the assemblies is checked by analyzing the assembly, assuming no interference between pin surfaces in relation to the holes, including position and size defects. This analysis is carried out in different steps corresponding to analysis of the assembly at different scales (between pairs of pins and holes and for every pin and hole). These four steps are:

- i. Computation of contact constraints of a pin / hole pair,

- ii. Computation of a contact polytope of a pin / hole pair,

- iii. Computation of the resulting polytope of the flange,

- iv. Detection of assembly interferences.

\subsubsection{Computation of contact constraints of a pin / hole pair}

Different approaches could be used to generate the contact constraints between pins and holes. In this study, we propose to simplify the expression of the constraints by discretizing of surfaces. To do this, the hole is discretized by $n_{d}$ points as shown in Fig. 5(b). The contact constraints of a pin / hole pair are then defined through a set of linear inequality constraints (Equation 1). These constraints guarantee non-interference between the pin and the hole. Thus, the displacement of the pin in relation to the hole $\left(t_{N_{O i}-1, i / 2, i}\right)$ has to be smaller than the local clearance divided by two. $t_{N_{O i}-1, i / 2, i}$ is the translation vector of pin $\mathrm{i}$ in relation to hole $\mathrm{i}$ at point $\mathrm{N}_{O i}$.

The accuracy of the polytope simulation is directly correlated to the number of points used for the discretization of pins and holes. It can be defined as the maximum distance between the boundary of the quadratic contact constraints and the boundary of the linear contact constraints. Theoretically, the quadratic contact constraints determines that the centre of a pin must lie in a circular zone (see Fig. 5(a)). The diameter of this circular zone is the difference between the diameters of the hole and the pin (i.e. clearance $c$ of the joint). The discretization induced by the polytopes approximates the circular zone by a polygonal zone (see Fig. 5(b)). This polygonal zone is inscribed in the circular zone, then the precision is the distance $e$, see Fig. 5(c). More details on the influence of the discretization are given in the work by Arroyave Tobon et al (2016).

$$
\begin{aligned}
& \forall i \in\{1, \ldots, 5\}, \forall j \in\left\{1, \ldots, n_{d}\right\}: \\
& t_{N_{0 i}-1, i / 2, i} \cdot \vec{n}_{j} \leq \frac{D_{2, i}-D_{1, i}}{2} \\
& \text { With: } \\
& \left\{\begin{array}{l}
D_{1, i} \leq D_{2, i} \\
\vec{n}_{j}=\cos \theta_{j} \cdot \vec{x}+\sin \theta_{j} \cdot \vec{y} \\
\theta_{j}=\frac{j}{n_{d}} \cdot 2 \pi \text { where } n_{d} \text { is the number of discretization points }
\end{array}\right.
\end{aligned}
$$

According to the Chasles theorem, $t_{N_{0 i}-1, i / 2, i}$ can be expressed as a function of local small displacement between the nominal position of the $i^{t h}$ pin and the $i^{t h}$ hole (called 


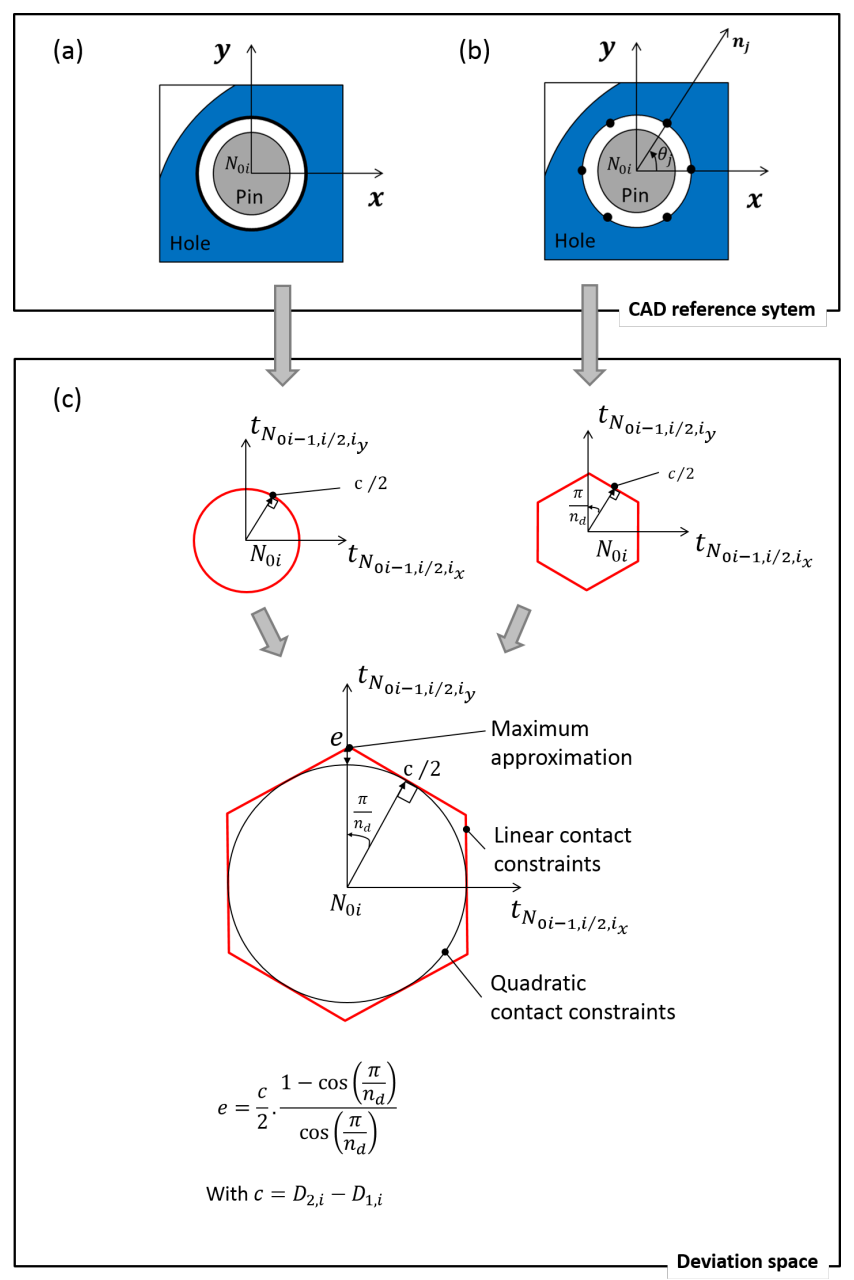

Figure 5: Influence of the discretization of contact constraints. (a) Quadratic contact constraints. (b) Linear contact constraints. (c) Approximation of the discretization. 
$\left.t_{N_{0 i}-1,0 / 2,0}\right)$, and the location defects of the pin and the hole $\left(e_{N_{0 i}-1, i / 1,0}\right.$ and $e_{N_{0 i}-2, i / 2,0}$ respectively):

$$
\begin{aligned}
& \forall i \in\{1, \ldots, 5\}, \\
& t_{N_{0 i}-1, i / 2, i}=e_{N_{0 i}-1, i / 1,0}+t_{N_{0 i}-1,0 / 2,0}-e_{N_{0 i}-2, i / 2,0}
\end{aligned}
$$

With :

- $e_{N_{0 i}-1, i / 1,0}$ : vector of location defect of pin $i$ in relation to its nominal location at point $N_{O i}$;

- $e_{N_{0 i}-2, i / 2,0}$ : vector of location defect of hole $i$ in relation to its nominal location at point $N_{O i}$;

- $t_{N_{0 i}-1,0 / 2,0}$ : translation vector of the nominal center of pin $i$ in relation to the nominal center of hole $i$ at point $N_{O i}$.

- $N_{O i}$ : nominal center of pin / hole pair i.

According to the small displacements field property (Bourdet et al, 1996; Clément and Bourdet, 1988), considering the possible displacement of the nominal center of part 1 in relation to the nominal center of part 2 defined by $t_{O-1,0 / 2,0}$, we could write:

$$
\begin{aligned}
& \forall i \in\{1, \ldots, 5\}, t_{N_{0 i}-1, i / 2, i}= \\
& e_{N_{0 i}-1, i / 1,0}+\left(t_{O-1,0 / 2,0}-O \vec{N}_{0 i} \times r_{1,0 / 2,0}\right)-e_{N_{0 i}-2, i / 2,0}
\end{aligned}
$$

With :

- $t_{O-1,0 / 2,0}$ : translation vector of the nominal center of pin $i$ in relation to the nominal center of hole $i$ at point $O$ along $\vec{x}$ and $\vec{y}$,

- $r_{1,0 / 2,0}$ : rotation vector of part 1 in relation to part 2 along the $\vec{z}$ axis,

- $O \vec{N}_{0 i}$ : position vector of point $\vec{N}_{0 i}$ in reference system $R_{0}$,

- $O$ : nominal center of the flange.

The projections of these vectors in the flange plane are:

- $e_{N_{0 i}-1, i / 1,0}=e_{N_{0 i}-1, i / 1,0_{x}} \cdot \vec{x}+e_{N_{0 i}-1, i / 1,0_{y}} \cdot \vec{y}$

- $e_{N_{0 i}-2, i / 2,0}=e_{N_{0 i}-2, i / 2,0_{x}} \cdot \vec{x}+e_{N_{0 i}-2, i / 2,0_{y}} \cdot \vec{y}$

- $t_{O-1,0 / 2,0}=t_{O-1,0 / 2,0_{x}} \cdot \vec{x}+t_{O-1,0 / 2,0_{y}} \cdot \vec{y}$

- $r_{1,0 / 2,0}=r_{1,0 / 2,0_{z}} \cdot \vec{z}$

- $O \vec{N}_{0 i}=R_{t h} \cdot \cos \theta_{t h_{i}} \cdot \vec{x}+R_{t h} \cdot \sin \theta_{t h_{i}} \cdot \vec{y}$ 
From equations 1 and 3, the contact constraints can be expressed at the center $O$ of the flange by the relation:

$$
\begin{aligned}
& \forall i \in\{1, \ldots, 5\}, \forall j \in\left\{1, \ldots, n_{d}\right\}: \\
& \left(e_{N_{0 i}-1, i / 1,0_{x}}+t_{O-1,0 / 2,0_{x}}-e_{N_{0 i}-2, i / 2,0_{x}}\right) \cdot \cos \theta_{j} \\
& +\left(e_{N_{0 i}-1, i / 1,0_{y}}+t_{O-1,0 / 2,0_{y}}-e_{N_{0 i}-2, i / 2,0_{y}}\right) \cdot \sin \theta_{j} \\
& +r_{1,0 / 2,0_{z}} \cdot R_{t h} \cdot \sin \left(\theta_{j}-\theta_{t h_{i}}\right) \leq \frac{D_{2, i}-D_{1, i}}{2}
\end{aligned}
$$

\subsubsection{Computation of a contact polytope of a pin / hole pair}

For a pin and hole pair $i$, we propose to compute $\bar{H}_{i, j}^{-}$corresponding to the $3-$ dimension half-space of the contact constraint derived from the $j^{\text {th }}$ discretization point expressed at the center $O$ of the flange.

$$
\begin{aligned}
& \forall i \in\{1, \ldots, 5\}, \forall j \in\left\{1, \ldots, n_{d}\right\}: \\
& \bar{H}_{i, j}^{-}=a_{i j 1} \cdot x_{1}+a_{i j 2} \cdot x_{2}+a_{i j 3} \cdot x_{3}+b_{i j} \leq 0 \\
& \text { With: } \\
& \left\{\begin{aligned}
a_{i j 1}= & R_{t h} \cdot \sin \left(\theta_{j}-\theta_{t h_{i}}\right) \text { and } x_{1}=r_{1,0 / 2,0_{z}} \\
a_{i j 2}= & \cos \theta_{j} \text { and } x_{2}=t_{O-1,0 / 2,0_{x}} \\
a_{i j 3}= & \sin \theta_{j} \text { and } x_{3}=t_{O-1,0 / 2,0_{y}} \\
b_{i j}= & \left(e_{N_{0 i}-1, i / 1,0_{x}}-e_{N_{0 i}-2, i / 2,0_{x}}\right) \cdot \cos \theta_{j} \\
& +\left(e_{N_{0 i}-1, i / 1,0_{y}}-e_{N_{0 i}-2, i / 2,0_{y}}\right) \cdot \sin \theta_{j} \\
& -\frac{D_{2, i}-D_{1, i}}{2}
\end{aligned}\right.
\end{aligned}
$$

$a_{i j 1}, a_{i j 2}$ and $a_{i j 3}$ come from the nominal definition of the parts and the discretization of the contact constraints.

The location and the diameter defects of the pins and the holes impact only the second member $b_{i j}$.

$x_{1}, x_{2}$ and $x_{3}$ are the unknowns of the problem. If there is at least one triplet that satisfies the system of inequalities in Equation 5, the parts can be assembled without interferences.

\subsubsection{Computation of the resulting polytope of the flange}

For a pin and hole pair $i$, the intersection of these half-spaces leads to the operand polytope expressed in Equation 6:

$$
\forall i \in\{1, \ldots, 5\}: P_{i}=\bigcap_{j=1}^{n_{d}} \bar{H}_{i, j}^{-}
$$

The operand polytope $P_{i}$ describes all the possible displacements of the pin $i$ in relation with the hole $i$ in the flange plane.

The resulting polytope of the flange, called $P_{R}$, comes from the intersection of the five operand polytopes $P_{i}$ computed by Equation 7:

$$
P_{R}=\bigcap_{i=1}^{5} P_{i}=\bigcap_{i=1}^{5}\left(\bigcap_{j=1}^{n_{d}} \bar{H}_{i, j}^{-}\right)
$$



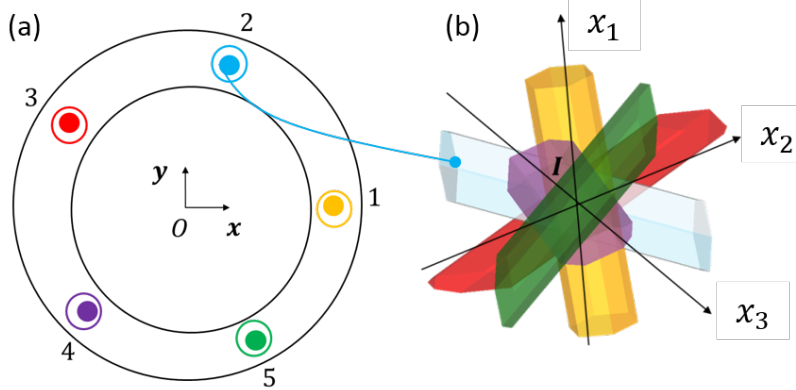

(c)

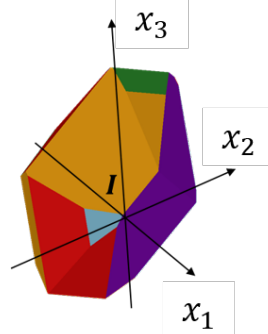

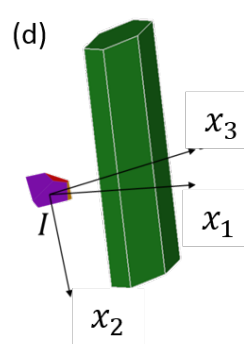

With $x_{1}=r_{1,0 / 2,0 z} ; x_{2}=t_{O-1,0 / 2,0_{x}} ; x_{3}=t_{O-1,0 / 2,0_{y}}$

Figure 6: Resulting polytope of a flange. (a) Drawing of the studied flange. (b) Pin / hole pairs polytopes. (c) Resulting polytope of a flange assembled without interference. (d) Empty intersection due to interferences

\subsubsection{Detection of assembly interferences}

The different results of the polytope computations are shown in Fig. 6 (a) and (b). According to the local gap and the location defects of the pins and the holes, $P_{i}$ can be computed. In the figure, a particular color was chosen for each pin / hole pair and the corresponding polytope is drawn with the same color.

The boundary of a polytope $P_{i}$ is the extrusion of a polygon.

The location of the polygon in the plane $\left(t_{O-1,0 / 2,0_{x}}, t_{O-1,0 / 2,0_{y}}\right)$ depends on the second member $b_{i j}$ of the halfspaces $\bar{H}_{i, j}^{-}$. $b_{i j}$ depends on the location defects of the pin and the hole i (see equation 5).

The direction of the extrusion is defined by the linear dependency between the rotation vector and the translation vector expressed at point $O$.

This linear dependency is defined by the coefficient $a_{i j}$ from equation 5. (If $a_{i j}=0$, then the direction of the extrusion is parallel to $r_{1,0 / 2,0_{z}}$ axis (i.e. $x_{1}$ axis)).

From the intersection of these polytopes, two extreme configurations can be seen. The first, shown in Fig. 6 (c), corresponds to a non-empty resultant polytope, $P_{R}$. In this case, parts 1 and 2 can be assembled without any interference. Thus, $P_{R}$ represents all 
the possible displacements between these two parts in the flange plane (the translations along the $\vec{x}$ and $\vec{y}$-axes, and the rotation along the $\vec{z}$-axis). This simulation is performed using the PolitoCAT and Politopix software tools, available on Open source (Delos and Teissandier, 2015). More details on polytope computations and uses are available in the recent studies by Arroyave Tobon et al (2016).

Concerning the configuration in Fig. 6 (d), the intersection between $P_{i}$ is null (leading to an empty $P_{R}$ ) resulting in the non-respect of at least one contact constraint. On the part geometry, one or several interferences could then be identified and the assembly cannot be possible.

\subsection{Synthesis of assembly simulations (rigid parts)}

From the definition of part defects (section 3.2), a stochastic simulation is executed by means of the Monte Carlo method and 10,000 pairs of parts with defects were generated. The part defects follow the normal distributions defined in Section 3.2. Based on the relations developed in the Section 3.3, we are able to check if the assembly is possible (i.e. without interference between pins and holes). The contact constraints have been discretized into nine points, which give a precision of $1 \mu \mathrm{m}$. From the assembly simulation, the compliance rate is then deduced and $99 \%$ of the assemblies are possible. To check if the amount of assembly used for the Monte Carlo simulation is enough according to the computed rate, the computation was re-run several times and the same rate was obtained.

Concerning the non-compliant assembly, it was observed that most of the time, four pins and holes could be assembled and only the assembly of the last pair is impossible due to interferences as shown in Fig. 7(a). The resulting polytopes can be seen in Fig. 6(d) for which the intersection between the first four pins and holes leads to the small polytope (in purple) and the other one, resulting from the last pin and hole pair, is drawn in green. Since, the polytopes are defined in displacement spaces (small translations along $\mathrm{x}$ and $\mathrm{y}$ axes and rotation along $\mathrm{z}$-axis respectively), it was possible to compute the minimal distance between these two polytopes for every assembly tested. This vector computation could be used to rank the difficulty to assemble the parts assuming deformations. With the developed computation, we are also able to clearly identify the minimal distance between points, faces and edges of the polytopes. From this, the minimal interference value is deduced, corresponding to the optimal position for the assembly. This distance in the geometric space (spatial coordinates where the parts are drawn), is determined with the CGAL QP-solver program (Schonherr, 2002). The computed minimal distance depends on the assembly sequence. In this instance we kept the configuration that gives the minimal interference value. More details on the diagnosis of assembly interferences are available in the works of Gouyou et al (2016). In the next section, this distance will be used as an interference value.

\section{Simulation of part deformations}

In the case of non-compliant assemblies, it was observed that the interference value is relatively small (few $\mu m$ ). In real conditions, part deformations may occur and the assembly could be possible. This phenomenon changes the initial hypothesis related to 
the part behavior (supposed to be rigid). Part deformations could be located in different zones and at different scales. On the one hand, local deformations may occur on the contact zone between pins and holes; on the other hand, the global shape of parts could be deformed by the assembly phase and could lead to a modification in the position and the shape of features.

\subsection{Developed approach}

To address such part behavior and to simulate the assembly phase, we propose to quantify the deformations by means of finite element simulations when the parts cannot be assembled without interference. Two parts were selected (including location and local gap of pins and holes) from the $1 \%$ of non-compliant assemblies obtained in Section 3.4. To do this, four different steps are required, as follows:

- i. Definition of the part geometries. For this step, the parts are positioned as shown in Fig. 3. The location and diameter defects of the pins and the holes (from Monte Carlo simulations) are introduced into the parts. Based on these hypotheses, the geometry is imported and meshed into the finite element code. The parts are governed by linear elastic and isotropic material behavior. At this point, only the contact between the two planar faces of the flange is defined;

- ii. Suppression of assembly interferences. In this step, displacements are set on the pins and the holes to place them at their nominal location. Since the diameter of a pin is always smaller than the diameter of a hole, there are no more assembly interferences; these displacements generate a strain and stress state in the parts;

- iii. Pin / hole contact activation. The displacements of the pins and the holes set in the previous step are deactivated and the contacts between the pins and the holes are turned on. The parts will then tend to return to their original geometry (set on point i). A steady state is then defined by the finite element simulation and it will result in local stress state between pins and holes and local pressure in contact zones;

- iv. Quantification of the difficulty level to make the assembly. The higher the pressure in the contact zones, the more difficult the assembly will be. To rank this difficulty, it is proposed at this step to quantify the minimal force needed to open the assembly. A tangential behavior is introduced following a Coulomb friction law. A small displacement (in this case equal to $2 \mu \mathrm{m}$ ) is imposed along the $\mathrm{z}$-axis on part 1 , keeping part 2 in the same position. The force needed is computed and enables to rank the difficulty level for making the assembly.

The finite element simulation was performed through Abaqus software with a static implicit formulation (Hibbitt et al, 2011). The assembly model is composed of 55,000 tetrahedral elements. To capture the evolution of the stress value due to local contacts between the pins and the holes, the mesh was densified in this zone. The steel material that makes up the two parts is only modeled by an elastic behavior $(E=210,000 \mathrm{MPa}$; $v=0.3)$. The deformations affect the local contacts between pins and holes and the 
global shape of the parts. Throughout the numerical simulation, the stress state in the part remains below the yield stress of the material $\left(R_{02 \%}=250 \mathrm{MPa}\right)$.

\subsection{Computations and results}

To quantify the influence of the interference value on the assembly of parts, three different scenarios were tested following the simulation conditions: pin / hole pairs 1 and 3 are in contact while pin 5 is in interference with hole 5 (see Fig 7); the other pairs are assembled with a gap condition. The value of local clearances between pins and holes is $30 \mu \mathrm{m}$. The locations of pins and holes, for the first scenario, are given in Tables 3 and 4. This configuration leads to an interference value of $5 \mu \mathrm{m}$ (Gouyou et al, 2016) (determined by computing the minimal distance between the polytopes as shown in Section 3.4). To increase this value, pin 5 is sequentially moved through two other positions in the same direction, drawn in blue in Fig. 7(a) (i.e. the minimal distance between the polytopes was increased). These three positions lead to various interferences values ranging from $5 \mu \mathrm{m}$ to $50 \mu \mathrm{m}$ (see configurations in Table 5).

During the deformation of parts, the contacts between pins and holes are modified according to the interference value. Fig. 7 illustrates this phenomenon. It corresponds to the last configuration of Table 5 (for an interference value of $50 \mu \mathrm{m}$ ). The initial contact areas between parts 1 and 2 are shown in Fig. 7(a). Pins 1 and 3 are in contact with their respective holes and pin 5 is in interference with hole 5. In Fig. 7(b), the part deformations have been computed following the finite element procedure described in Section 4.1. It can be seen that the contacts have changed drastically. In the final position, only the $2^{\text {nd }}$ pin / hole pair remains without contact. These effective local contacts lead to local pressure in the contact zones. Added to these deformations, a small displacement of one part compared to the other has been observed and corresponds to a small rigid displacement of a body part.

From the local pressure value and according to the size of contact mesh, it is proposed to compute an equivalent local force. From this value, assuming a friction coefficient between parts governed by Coulomb friction law with a value of 0.1 , the load needded to finalize the assembly between parts can be deduced. All values are listed in Table 5 and for the last configuration, the assembly load is equal to $450 \mathrm{~N}$.

For the first configuration, with a very small interference value of $5 \mu \mathrm{m}$, the corresponding force is equal to $10 \mathrm{~N}$. This value is very small and validates the accuracy of the finite element computation. It can be observed, that the evolution of the assembly load is not linear. For example, from 5 to $20 \mu \mathrm{m}$, the load changes from 10 to $100 \mathrm{~N}$. If the interference value is equal to $50 \mu \mathrm{m}$, the corresponding load is $450 \mathrm{~N}$. This load behavior is the result of modifying of contacts between pins and holes during the deformation of parts.

\subsection{Discussion}

The major contribution of this paper is to address overconstrained assembly with uncertain conditions of interference between parts (depending on the part defects, the parts can be assembled either with gap or with interferences). The first step is to determine if 
Table 3: Positions of pins

\begin{tabular}{llllll}
\hline & Pos. Pin 1 & Pos. Pin 2 & Pos. Pin 3 & Pos. Pin 4 & Pos. Pin 5 \\
\hline $\mathrm{x}$ & 200.009 & 61.804 & -161.805 & -161.807 & 61.808 \\
\hline $\mathrm{y}$ & 0.00 & 190.216 & 117.572 & -117.560 & -190.235 \\
\hline
\end{tabular}

Table 4: Positions of holes

\begin{tabular}{llllll}
\hline & Pos. Hole 1 & Pos. Hole 2 & Pos. Hole 3 & Pos. Hole 4 & Pos. Hole 5 \\
\hline $\mathrm{x}$ & 200.001 & 61.800 & -161.801 & -161.805 & 61.803 \\
\hline $\mathrm{y}$ & 0.001 & 190.212 & 117.558 & -117.558 & -190.211 \\
\hline
\end{tabular}

Table 5: Tested positions for the pin 5, associated interference values and computation results (the other locations of pins are defined in table 3)

\begin{tabular}{|c|c|c|c|c|}
\hline \# & & $\begin{array}{l}\text { Pos. } \\
\text { Pin } 5\end{array}$ & $\begin{array}{l}\text { Interference } \\
(\mu m)\end{array}$ & $\begin{array}{l}\text { Assembly } \\
\text { Force }(\mathrm{N})\end{array}$ \\
\hline \multirow{2}{*}{1} & $\mathrm{X}$ & 61.808 & 5 & 10 \\
\hline & y & -190.235 & & \\
\hline \multirow{2}{*}{2} & $\mathrm{x}$ & 61.816 & 20 & 100 \\
\hline & $y$ & -190.248 & & \\
\hline \multirow{2}{*}{3} & $\mathrm{x}$ & 61.831 & 50 & 450 \\
\hline & $\mathrm{y}$ & -190.274 & & \\
\hline
\end{tabular}
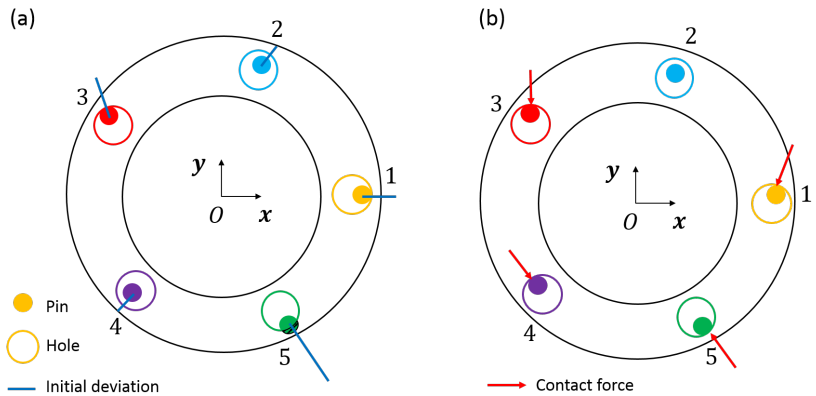

Figure 7: Illustration of the assembly deformation for the $3^{\text {rd }}$ configuration of the table 5. (a) before part deformations. (b) after part deformations. 
the assembly is possible without interference by means of the polytope computations. The classical hypotheses of the tolerance study are assumed. In the case of interference value, a typical procedure is defined based on a finite element simulation in which location defects and gaps between holes and pins are included. From this computation, we are able to quantify the difficulty level to make the assembly.

The deformations and the modifications to contact conditions lead to a rigid displacement of one part in relation to the other. In the assembly, it will be relevant to limit these deviations to ensure precision. Some modifications could be made to the technical drawing available in Fig. 2, by adding other functional specifications to limit the misalignment between parts. These new functional requirements lead to minor modifications of the tolerance problem (addition of inequality to respect) but the global procedure of the polytope construction will remain the same and could be carried out in future work.

More effort could also be made to validate experimentally the numerical procedure for identifying interferences and their locations, and the global deformation of parts. For the first point, measurement of parts will be required and measured defects of parts could be used to assemble the part numerically through the polytope approach. In the case of relatively large interference values, the real assembly of the part will be difficult and could validate the procedure. For the validation of part deformations, it seems to be more complex to validate the procedure since the interference value should be relatively small to ensure the possibility of assembling the parts. According to the authors, it will be more relevant to measure the global deformation of parts due to the assembly by optical means for example as proposed by Tyson et al (2002) or Pottier et al (2011) and to make a comparison between deformations computed by the finite element simulation and the measurements.

\section{Conclusion}

The geometrical analysis of an overconstrained mechanism is not an easy task mainly due to the complexity of mathematical tools and methods to deploy. Basically, many studies tend to simplify the architecture, making it isostatic (suppression of redundancies in the assembly).

In such cases, different software supports can be used to assist the designers in the tolerance analysis and computations. For more complex architectures, the overconstrained property should be addressed and particular methods and numerical tools have to be used. The present method is dedicated to the study of such overconstrained architecture composed of a planar pair joint added to five pin and hole contacts. It was decided to study the possibility of making the assembly of the two parts without any interference between parts. The tolerance analysis is mainly based on the polytope approach where the integration of geometric conditions between parts is expressed as a set of inequalities to respect. The part defect is derived from analysis of the production of the manufactured part. From the production characteristics, it is possible to identify non-compliant assemblies (i.e. assemblies where interference between parts has been identified). According to the polytope analysis, we found that the interference values are relatively small and it is possible that the part deformations can enable the parts to 
conform so that the assembly is possible. To check this property, a numerical procedure based on finite element simulations is set up. The ability to assemble the part is ranked by quantifying the effort required for mounting parts. Different values of interferences have been tested.

From the results, different mechanism behaviors could be identified as a function of the interference value. For small values, only local deformations occur between pins and holes and the contact between part and global position of one part compared to the other one remain the same.

For greater interference values, a competition between local deformations and the global part deformation is observed, depending on their relative stiffness. When the part deformation is activated, a drastic modification to the contact conditions is observed leading to greater displacement of parts. These modifications could impact other functional requirements such as precision mounting problems. This last point has not been considered in this study but will be the subject of a first extension to this work.

Next, in order to simplify the part deformation procedure, a mixed computation approach can be defined for the two scales of deformation (i.e. local and global). The nature of the local contacts between pins and holes makes it possible to use an analytical model governed by Hertz models, for example. For the global part deformation, it seems to be possible to make a numerical simplification using the super-element method. Part deformation is determined directly by defining a limit condition of a few key points located at the position of the mechanical joints and then the final deformation on effort required to assemble the parts is deduced. This operation will greatly reduce the computation time required for the assembly procedure assuming part deformations. More work will need to be carried out before these considerations can be implemented.

Finally, it is crucial to validate the results of simulations experimentally with the different assumptions (rigid parts or compliant parts). This process is currently under development for sets of parts defects.

\section{References}

Ameta G, Davidson JK, Shah JJ (2007) Using Tolerance-Maps to Generate Frequency Distributions of Clearance and Allocate Tolerances for Pin-Hole Assemblies. Journal of Computing and Information Science in Engineering 7(4):347-359

Ameta G, Samper S, Giordano M (2011) Comparison of Spatial Math Models for Tolerance Analysis: Tolerance-Maps, Deviation Domain, and TTRS. Journal of Computing and Information Science in Engineering 11:1-8

Anselmetti B (2010) CLIC: A Method for Geometric Specification of Products. In: Geometric tolerancing of products, ISBN 978-1-84821-118-6, ISTE-WILEY, pp 210239

Arroyave Tobon S, Teissandier D, Delos V (2016) Tolerance analysis with polytopes in HV-description. IDETC201659027, Charlotte (North Carolina - USA) 
Beaucaire P, Gayton N, Duc E, Dantan JY (2013) Statistical tolerance analysis of overconstrained mechanisms with gaps using system reliability methods. ComputerAided Design 45(12):1547-1555, DOI 10.1016/j.cad.2013.06.011

Bourdet P, Mathieu L, Lartigue C, Ballu A (1996) The concept of the small displacement torsor in metrology. Series on Advances in Mathematics for Applied Sciences, Advanced mathematical tools in metrology II 40:110-122

Breteau P, Thiebaut F, Lartigue C, Fricro B, Falgarone H, Moufle GE (2007) Assembly Simulation of Flexible Parts through the fitting of Linkage Devices. In: 10th CIRP conference on Computer Aided Tolerancing, Erlangen, Germany

Chevassus N, Falgarone H, Thiebaut F, Bourdet P, Moufle GE (2006) A New Approach for Best Fit Assembly Based on the Behaviour of Components

Cid G, Thiebaut F, Bourdet P, Falgarone H (2007) Geometrical Study of Assembly Behaviour, Taking into Accounts Rigid Components Deviations, Actual Geometric Variations and Deformations. In: Davidson JK (ed) Models for Computer Aided Tolerancing in Design and Manufacturing, Springer Netherlands, Dordrecht, pp 301310

Clément A, Bourdet P (1988) A study of optimal-criteria identification based on the small-displacement screw model. Annals of the CIRP 37(1)

Dantan JY, Qureshi AJ (2009) Worst-case and statistical tolerance analysis based on quantified constraint satisfaction problems and Monte Carlo simulation. ComputerAided Design 41(1):1-12, DOI 10.1016/j.cad.2008.11.003

Davidson JK, Mujezinovic A, Shah JJ (2002) A new mathematical model for geometric tolerances as applied to round faces. ASME Transactions on Journal of Mechanical Design 124:609-622

DCS (2011) DCS - Tolerance Analysis, GD\&T, and Quality Solutions, www.3dcs.com

Delos V, Teissandier D (2015) PolitoCAT and Politopix, http://i2m.ubordeaux.fr/politopix.html. URL http://i2m.u-bordeaux.fr/politopix.html

Fleming A (1988) Geometric relationships between toleranced features. Artificial Intelligence 37(1-3):403-412

Giordano M, Duret D, Tichadou S, Arrieux R (1992) Clearance space in volumic dimensioning. Annals of the CIRP 41(1):565-568

Gouyou D, Teissandier D, Delos V (2016) Tolerance Analysis by Polytopes: Application to Assembly Interferences Diagnosis. 14th CIRP CAT 2016 CIRP Conference on Computer Aided Tolerancing, vol 43, pp 52-57, DOI 10.1016/j.procir.2016.02.019

Hibbitt D, Karlsson B, Sorensen P (2011) ABAQUS 6.11: a computer software for finite element analysis 
Hu JS, Camelio J (2006) Modeling and control compliant assembly systems. Annals of the CIRP Manuf Technol 55(1):19-22

ISO5459 (2011) Geometrical tolerancing - Datums and datum systems

Jaishankar LN, Davidson JK, Shah JJ (2012) Representing Stresses That Arise in Parallel Assemblies That Contain Imperfect Geometry Allowed by Tolerances. ASME, pp 577-584, DOI 10.1115/DETC2012-70208

Jaishankar LN, Davidson JK, Shah JJ (2013) Tolerance Analysis of Parallel Assemblies Using Tolerance-Maps and a Functional Map Derived From Induced Deformations. ASME, DOI 10.1115/DETC2013-12394

Ledoux Y, Teissandier D (2013) Geometric variability analysis by a fiabilist approach : application to a turbine. Research In Engineering Design 24(3):297-311

Mansuy M, Giordano M, Davidson JK (2013a) Comparison of Two Similar Mathematical Models for Tolerance Analysis: T-Map and Deviation Domain. Journal of Mechanical Design 135(101008-1/101008-7)

Mansuy M, Giordano M, Hernandez P (2013b) A Generic Method for the Worst Case and Statistical Tridimensional Tolerancing Analysis. Procedia CIRP 10:276-282

MECAmaster SARL (2012) MECAmaster Assembly Software, www.mecamaster.com

Mounaud M, Thiebaut F, Bourdet P, Falgarone H, Chevassus N (2007) Integrating the flexibility of components in the assembly of aeronautics hydraulic systems. In: 10th CIRP conference on Computer Aided Tolerancing, Erlangen, Germany

Nigam SD, Turner JU (1995) Review of statistical approaches to tolerance analysis. Computer-Aided Design 27(1):6-15

Pezzuti E, Piscopo G, Ubertini A, Valentini PP (2005) Investigation on pin-hole connection in flexible assembly

Pierre L, Teissandier D, Nadeau JP (2014) Variational tolerancing analysis taking thermomechanical strains into account: Application to a high pressure turbine. Mechanism and Machine Theory 74:82-101, DOI 10.1016/j.mechmachtheory.2013.11.014

Pottier T, Toussaint F, Vacher P (2011) Contribution of heterogeneous strain field measurements and boundary conditions modelling in inverse identification of material parameters. European Journal of Mechanics - A/Solids 30(3):373-382

Samper S, Giordano M (2003) Simultaneous analysis method for tolerancing flexible mechanisms. Book Geometric Product Specification and Verification: Integration of Functionality pp 127-134

Schonherr S (2002) Quadratic Programming in Geometric Optimization: Theory, Implementation and Applications. $\mathrm{PhD}$ thesis, École polytechnique fédérale de Lausanne 
Shah JJ, Ameta G, Shen Z, Davidson JK (2007) Navigating the tolerance analysis maze. Computer-Aided Design and Applications 4(5):705-718

Shiu BW, Apley DW, Ceglarek D, Shi J (2003) Tolerance allocation for compliant beam structure assemblies. IIE Transactions 35(4):329-342

Soderberg R, Lindkvist L, Dahlstrm S (2006) Computer-aided robustness analysis for compliant assemblies. Journal of Engineering Design 17:411-428

Teissandier D, Delos V, Coutard Y (1999) Operations on polytopes: application to tolerance analysis. In: Global Consistency of Tolerances, ISBN 0-7923-5654-3, Kluwer academic publisher, Enschede (Netherlands), pp 425-433

Turner JU (1993) A Feasibility Space Approach for Automated Tolerancing. Journal of Engineering for Industry 115:341-346

Tyson J, Schmidt T, Galanulis K (2002) Biomechanics deformation and strain measurements with $3 \mathrm{~d}$ image correlation photogrammetry. Experimental Techniques 26(5):39-42

Xie K, Wells L, Camelio JA, Young BD (2007) Variation propagation analysis on compliant assemblies considering contact interaction. Journal of Manufacturing Science and Engineering 129(5):934-942 